\title{
Aspects actuels des infections nosocomiales au Centre Hospitalier Libanais de Beyrouth
}

\author{
A. Al-Hajje, ${ }^{1}$ M. Ezedine, ${ }^{2}$ H. Hammoud, ${ }^{3}$ S. Awada, ${ }^{1}$ S. Rachidi, ${ }^{1}$ S. Zein ${ }^{1}$ et P. Salameh ${ }^{1}$
}

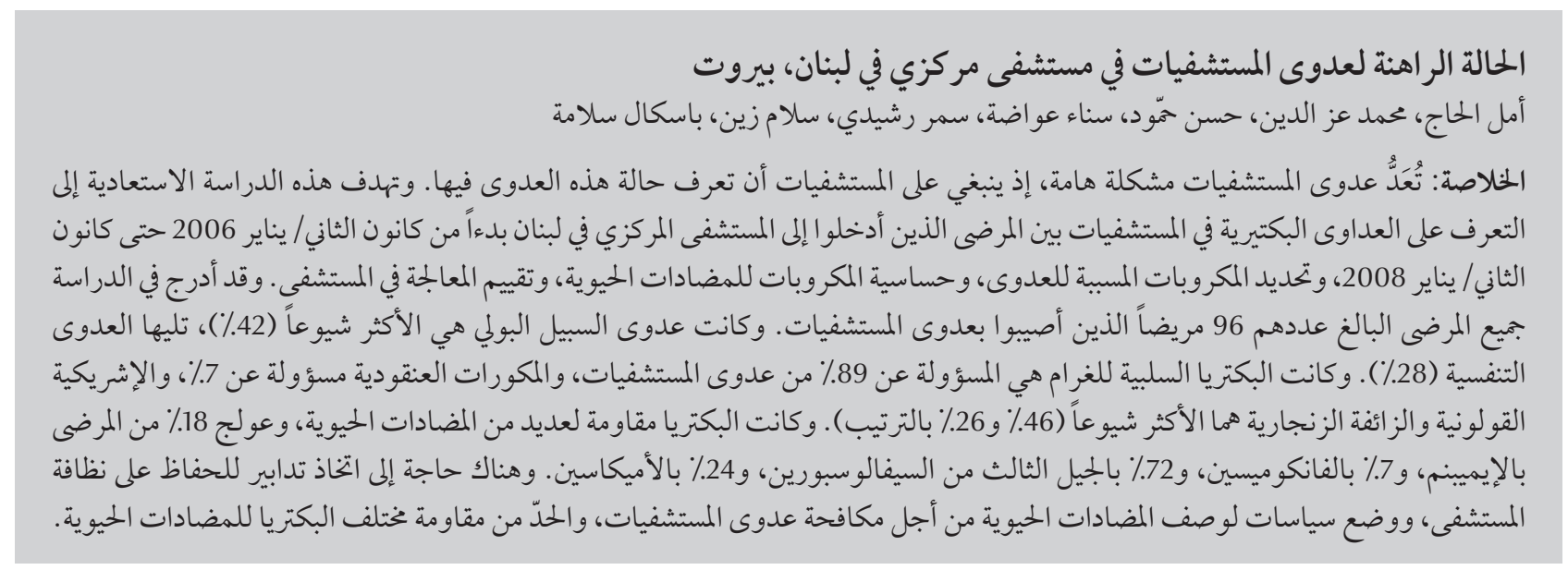

RÉSUMÉ Les infections nosocomiales constituent un problème majeur et tout hôpital doit connaître sa situation en matière d'infections nosocomiales. Cette étude rétrospective visait à identifier les infections nosocomiales bactériennes chez les patients admis au Centre Hospitalier Libanais de janvier 2006 à janvier 2008, déterminer les germes en cause, étudier la sensibilité de ces germes aux antibiotiques et évaluer le traitement adopté à l'hôpital. Au total, 96 patients développant une infection nosocomiale ont été recensés. Les infections nosocomiales les plus courantes étaient les infections urinaires (42\%), suivies par les infections pulmonaires (28 \%). Les bacilles à Gram négatif étaient responsables dans $89 \%$ des cas et les staphylocoques dans $7 \%$, Escherichia coli et Pseudomonas aeruginosa étant les plus fréquents (46\% et $26 \%$ respectivement), avec une résistance majeure à divers antibiotiques; $18 \%$ des patients infectés étaient traités par imipénème, $7 \%$ par vancomycine, $42 \%$ par céphalosporines de $3^{\text {e }}$ génération et $24 \%$ par amikacine. La lutte contre les infections nosocomiales doit passer par des mesures d'hygiène dans I'hôpital et par une politique de prescription d'antibiotiques adaptée à l'évolution des résistances bactériennes.

\section{Current status of nosocomial infections in the Lebanese Hospital Center, Beirut}

ABSTRACT Nosocomial infections are a significant problem and hospitals need to be aware of their nosocomial infection status. This retrospective study aimed to identify nosocomial bacterial infections in patients admitted to the Lebanese Hospital Center from January 2006 to January 2008 and determine the causative micro-organisms, the antibiotic sensitivity of the micro-organisms and evaluate the hospital treatment. In total 96 patients with nosocomial infection were included. Urinary infections were the commonest nosocomial infections (42\%) followed by pulmonary infections (28\%). Gram-negative bacteria were responsible for $89 \%$ of nosocomial infections and staphylococci for $7 \%$, with Esherichia coli and Pseudomonas aeruginosa being the most common (46\% and 26\% respectively) The organisms were resistant to multiples antibiotics and $18 \%$ of the patients were treated with imipenem, $7 \%$ with vancomycin, $42 \%$ with third-generation cephalosporins and $24 \%$ with amikacin. Hospital hygiene measures and antibiotic prescription policies are required to fight nosocomial infections and reduce antibiotic resistance among organisms. 


\section{Introduction}

Lesinfections nosocomiales constituent un problème réel de santé publique du fait de leur fréquence croissante, de leur gravité compte tenu de la multirésistance des germes en cause et de leur coût socio-économique. Si le temps des grandes épidémies est passé, ces infections touchent toujours les malades hospitalisés dans une proportion souvent inquiétante. Selon les données de la littérature, 5 à $10 \%$ des malades hospitalisés contractent une infection nosocomiale lors de leur séjour hospitalier [1].

Les infections nosocomiales sont définies comme des infections apparaissant au cours ou à la suite d'une hospitalisation et n'étant pas présentes, ni en incubation ni à l'admission à l'hôpital. Lorsque l'état infectieux à l'admission n'est pas connu, un délai d'au moins 48 heures après l'admission (ou un délai supérieur à la période d'incubation lorsque celleci est connue) est communément accepté pour distinguer une infection d'acquisition nosocomiale d'une infection communautaire $[2,3]$. Les infections nosocomiales peuvent concerner tous les types d'agents infectieux, mais elles sont le plus fréquemment bactériennes, et plus occasionnellement virales, fongiques ou parasitaires [2].

De nombreux facteurs contribuent à augmenter les risques d'acquérir ces infections, dont ceux liés au patient (âge avancé, immunodépression, diabète, obésité et dénutrition), ceux liés aux soins et aux interventions (sondage urinaire, gastrique ou trachéal, cathéter veineux, intervention chirurgicale, endoscopie) et ceux liés à l'agent infectieux (virulence et résistance aux antibiotiques) [4].

Notre étude, menée dans le Centre HospitalierLibanais (CHL), comprend les objectifs suivants : identifier les infections nosocomiales bactériennes chezles patients hospitalisés, dégager les caractéristiques des patients présentant ces infections nosocomiales, déterminer les germes en cause, étudier la sensibilité de ces germes aux antibiotiques et évaluer le traitement adopté à l'hôpital.

\section{Méthodes}

Nous avons mené une étude rétrospective sur une période de deux ans (janvier 2006-janvier 2008), incluant les patients hospitalisés dans les différents services du CHL et ayant présenté des infections nosocomiales bactériennes. Ont été exclus les patients développant une infection nosocomiale autre que bactérienne, les patients venant d'un autre hôpital, ceux présentant une infection à l'admission, ainsi que les services pédiatriques.

La sélection des patients ayant développé des infections nosocomiales était réalisée par la revue des rapports d'infections nosocomiales détectées par le CLIN (Comité de lutte contre les infections nosocomiales). Celui-ci considère une infection comme nosocomiale si son délai d'apparition est d'au moins 48 heures après l'admission $[2,3]$. Le support d'évaluation était un questionnaire rempli à partir des dossiers des malades ayant présenté ces infections, et constitué de trois parties:

- la partie «Caractéristiques des patients » comprenait l'âge, le sexe et les antécédents médicaux des patients;

- la partie «Culture bactérienne» décrivait le type d'examen bactériologique réalisé, le germe responsable, l'antibiogramme, la sensibilité et la résistance du germe aux antibiotiques étudiés. Les différents examens bactériologiques étudiés étaient l'hémoculture, l'examen cytobactériologique des urines (ECBU), les sécrétions pulmonaires par aspiration trachéale profonde et le pus.
- Lapartie «Traitementantibiotique » évaluait le traitement antibiotique empirique, le traitement antibiotique après réalisation de l'antibiogramme ainsi que la conformité de l'antibiotique eu égard à la dose et à la durée du traitement par rapport aux recommandations du Collège des Universitaires des Maladies Infectieuses et Tropicales (CMIT, exAPPIT - Association des professeurs de pathologies infectieuses et tropicales) et de la Commission des anti-infectieux de l'Agence régionale de l'hospitalisation (ARH) de Bourgogne $[5,6]$. Le profil de l'antibiogramme a été pris en compte selon la méthode des disques et a été interprétéselon les recommandations de la Société Française de Microbiologie (SFM) [7].

\section{Analyse statistique}

Nous avons mené une étude descriptive des différentes variables des patients ayant développé des infections nosocomiales, des germes en cause et du traitement antibiotique. Les calculs statistiques ont été réalisés à l'aide du logiciel SPSS.

\section{Résultats}

Quatre-vingt-seize patients hospitalisés développant une infection nosocomiale ont été inclus dans notre étude. Il s'agissait de 50 hommes et 46 femmes. L'âge moyen de notre population était de 64 ans ; $69 \%$ avaient un âge supérieur ou égal à 65 ans. Soixante-dix-huit pour cent $(78 \%)$ des patients avaient au moins deux antécédents médicaux; parmi ces antécédents figuraient les maladies cardiaques (68\%) et le diabète (46\%). Les 96 patients de notre étude ne présentaient aucune infection à leur admission. La durée moyenne d'hospitalisation était de 10 jours. Le tableau 1 synthétise les différentes caractéristiques des patients ayant développé des infections nosocomiales. 


\begin{tabular}{lcc}
\hline $\begin{array}{l}\text { Tableau 1 Caractéristiques des patients ayant développé des infections } \\
\text { nosocomiales }\end{array}$ & $\begin{array}{c}\text { Nbre } \\
(\mathbf{n}=\mathbf{9 6})\end{array}$ & $\%$ \\
\hline Caractéristiques & 66 & 69 \\
Âge $\geq 65$ ans & 50 & 52 \\
Sexe masculin & 65 & 68 \\
Antécédents de maladies cardiaques & 44 & 46 \\
Antécédents de diabète & 30 & 31 \\
Antécédents de chirurgie (de plus d'1 an) & 22 & 23 \\
Antécédents d'insuffisance rénale & 13 & 14 \\
Antécédents de maladies respiratoires & 8 & 8 \\
Antécédents d'insuffisance hépatique & & \\
\hline
\end{tabular}

Soixante-douze pour cent (72\%) des patients porteurs d'infections nosocomiales étaient hospitalisés dans les services de soins intensifs, $19 \%$ en médecine interne et $9 \%$ en chirurgie.

Les localisations étaient surtout urinaires $(42 \%)$ et pulmonaires (28\%). Suivaient les bactériémies (19\%) et les infections nosocomiales de plaie opératoire ( $8 \%)$. D'autres localisations étaient retrouvées, notamment cutanées (3\%).

Pour les infections pulmonaires, la porte d'entrée ou le facteur d'exposition était la sonde naso-gastrique ou trachéo-bronchique, alors que pour les infections nosocomiales urinaires, c'était la sonde urinaire que portaient presque la totalité des patients. Les infections de plaie opératoire étaient superficielles.

Quatre-vingt-neufpour cent (89\%) des germes isolés étaient des bacilles à Gram négatif avec une prédominance d'Escherichia coli (46\%), suivi du germe Pseudomonas aeruginosa (26\%). Le tableau 2 présente les différentes bactéries isolées.

Concernant les infections urinaires, les souches nosocomiales d'E. coli représentaient la proportion la plus élevée et pourlesinfections pulmonaires, les $P$. aeruginosa venaient au premier rang. Staphylococcus aureus était retrouvé dans des proportions non négligeables, avec un taux de $7 \%$ de bactéries isolées. Ces germes partageaient presque la même fréquence entre les bactériémies et les infections nosocomiales de plaie opératoire ( $4 \%$ et $3 \%$ ).

En ce qui concerne la sensibilité aux antibiotiques des germes isolés, la totalité des souches d'E. coli étaient résistantes aux céphalosporines de deuxième génération et environ la moitié à l'association amoxicillineacide clavulanique. Toutes les souches d'E. coli étaient sensibles aux céphalosporines de troisième génération et aux aminosides. Seule

\begin{tabular}{lcc}
\hline Tableau 2 Distribution des infections nosocomiales par germes isolés & \\
\hline Germes isolés & $\begin{array}{c}\text { Nbre } \\
(\mathbf{n}=\mathbf{9 6})\end{array}$ & $\%$ \\
\hline Escherichia coli & 44 & 46 \\
Pseudomonas aeruginosa & 25 & 26 \\
Klebsiella spp. & 11 & 11 \\
Staphylococcus aureus & 7 & 7 \\
Acinetobacter spp. & 4 & 4 \\
Proteus mirabilis & 1 & 1 \\
Autres & 4 & 4 \\
\hline
\end{tabular}

ment $20 \%$ des souches de Klebsiella étaient résistantes aux céphalosporines de troisième génération. La totalité des souches de $S$. aureus étaient résistantes aux céphalosporines de deuxième génération et le caractère méticillino-résistant (méti-R) était trouvé dans $72 \%$ des souches. Toutes les infections à $S$. aureus étaient traitées par vancomycine.

Près de $65 \%$ des patients infectés étaient traités par imipénème dès qu'une infection était détectée. Mais selon le résultat de l'antibiogramme, l'imipénème était administré chez $18 \%$ des patients ayant développé des infections nosocomiales, les céphalosporines de troisième génération chez $42 \%$, l'amikacine chez $24 \%$ et la vancomycine chez $7 \%$ (Tableau 3).

\section{Discussion}

Les infections nosocomiales présentes dans nos structures de soins constituent un problème majeur de santé publique. Dans notre étude, les patients âgés de 65 ans et plus paraissaient plus exposés auxinfections nosocomiales, confirmant le fait que l'âge avancé constitue un facteur de risque de développer une infection nosocomiale $[1,4]$. Ce n'est sans doute pas l'âge seulement, mais plutôt la pathologie sous-jacente fréquente chez les patients âgés qui affaiblit leurs défenses et les rend plus exposés aux risques infectieux [1]. Les pathologies responsables d'une sensibilité particulière à certaines infections (pathologie chronique, qu'elle soit cardio-vasculaire, rénale, respiratoire ou hépatique, dénutrition, diabète, etc.) paraissent comme des facteurs de risque propres aux malades et déterminent en grande partie la fréquence des infections nosocomiales [1]. Dans notre étude, $78 \%$ des patients présentaient au moins deux antécédents médicaux. 


\begin{tabular}{lcc}
\hline $\begin{array}{l}\text { Tableau } 3 \text { Fréquence des antibiotiques utilisés après les résultats de } \\
\text { l'antibiogramme }\end{array}$ & $\begin{array}{c}\text { Nbre } \\
(\mathbf{n}=\mathbf{9 6})\end{array}$ & $\%$ \\
\hline Antibiotiques & 40 & 42 \\
Céphalosporines de troisième génération & 18 & 19 \\
Ceftazidine & 12 & 13 \\
Ceftriaxone & 10 & 10 \\
Cefotaxime & 23 & 24 \\
Amikacine & 17 & 18 \\
Imipénème & 9 & 9 \\
Lévofloxacine & 7 & 7 \\
Vancomycine & 7 \\
\hline
\end{tabular}

La prévalence des infections nosocomiales pour un service donné varie selon la durée d'hospitalisation mais aussi selon les activités qui y sont menées : $72 \%$ des patients porteurs d'infections nosocomiales étaient retrouvés dans les services de soins intensifs. Nos résultats sont comparables à d'autres enquêtes $[1,8]$. Les taux élevés observés dans les services de soins intensifs pourraient être en rapport avec la sévérité des pathologies sous-jacentes, le séjour relativement prolongé des patients et la fréquence des procédures invasives à visée diagnostique et/ou thérapeutique [9-11].

Les infections urinaires étaient prédominantes, ce qui est généralement le cas dans d'autres études [12-14]; cela peut être expliqué par l'utilisation courante des bandelettes urinaires et la demandequasi systématique del'ECBU dans certains pays, alors que dans notre étude c'est par la présence de sondes urinaires.Le sondage urinaire représente l'un des principaux gestes de soins à risque d'infection nosocomiale [10]. La pose de sonde urinaire constitue le facteur de risque majeur des infections urinaires et exige qu'elle soit un acte médical [15]. L'évaluation des pratiques de sondage et la mise en place de mesures pour diminuer ce risque sont nécessaires [16]. Ces actions vont de la suppression des sondes inutiles à la limitation des sondes exploratrices et l'introduction du système de sonde clos [17].

Les infections pulmonaires étaient retrouvées fréquemment dans notre étude (28\%) du fait du risque infectieux élevé chez les patients en soins intensifs portant des sondes naso-gastriques ou trachéo-bronchiques. La ventilation artificielle constitue le principal facteur de risque dans la survenue des infections pulmonaires nosocomiales $[9,18,19]$. La prévention de ces infections repose avant tout sur la maitrise des risques évitables et vise essentiellement la réanimation et les soins intensifs. Elle consiste souvent en une désinfection et une stérilisation soigneuse des appareils de ventilation, des aspirateurs et des nébuliseurs [18]. L’alimentation entérale a encore été identifiée comme facteur de risque significatif dans la littérature. Une étude mexicaine montre qu'elle expose les malades à un risque accru d'infection nosocomiale [9].

Les infections nosocomiales de plaie opératoire venaient en quatrième place $(8 \%)$. Le risque nosocomial lié à l'infection du site opératoire n'est pas toujours élevé [20]. Il est en rapport avec les caractéristiques préalables du patient, la durée et le type d'intervention [21]. Pour lutter contre ces infections, des mesures préventives spécifiques doivent s'appliquer en pré-opératoire, au bloc et durant la période post-opératoire [3].

Dans notre étude, les bacilles à Gram négatif étaient les germes les plus fréquemment retrouvés comme agents responsables des infections nosocomiales avec une prédominance d'E. coli, ce qui est retrouvé également dans la plupart des séries bibliographiques [1214]. Les souches nosocomiales de $P$. aeruginosa étaient rencontrées avec une fréquence importante dans notre étude et venaient au premier rang dans les infections pulmonaires. Ces germes sont prédominants dans les infections pulmonaires $[3,6]$.

La totalité des souches d'E. coli étaient résistantes aux céphalosporines de deuxième génération et environ la moitié à l'association amoxicillineacide clavulanique. La conduite thérapeutique devant une bactérie résistante est d'utiliser une association d'antibiotiques bactéricides à spectre étroit pour réduire la possibilité de sélection de mutants encore plus résistants [22].

S. aureus, retrouvé avec un taux de $7 \%$, était principalement rencontré dans les bactériémies et les infections de plaie opératoire. Ceci est dû à la multiplication des différents gestes invasifs [23]. Ces germes sont montrés insensibles aux céphalosporines de deuxième génération et à l'association amoxicilline-acide clavulanique, conformément aux données de la littérature indiquant que ces germes sont généralement insensibles aux $\beta$-lactamines [12]. Dans notre étude, tous les germes isolés de $S$. aureus étaient traités par vancomycine malgré la sensibilité de ce germe à la méticilline dans $28 \%$ des cas.

En ce qui concerne le germe $P$. aeruginosa, mis à part sa résistance naturelle vis-à-vis des céphalosporines de première génération et de l'amoxicilline-acide clavulanique (Augmentin), ce bacille a une forte propension à produire de nouvelles souches résistantes aux antibiotiques sélectionnés en milieu hospitalier. Dans notre étude, $68 \%$ des souches de $P$. aeruginosa étaient 
résistantes à l'imipénème. Cette résistance peut être expliquée par la perte de la porine D2 qui entraîne une diminution spécifique de la perméabilité pour l'imipénème. La résistance ne s'exprime à haut niveau que si la perte de D2 est accompagnée d'une hyperproduction de la céphalosporinase chromosomique [24]. Les souches de $P$. aeruginosa peuvent avoir encore une tendance à acquérir des plasmides qui leur confèrent un haut degré de résistance par exemple à la gentamycine et à la tobramycine [25]. Dans notre étude, toutes les souches nosocomiales de $P$. aeruginosa étaient sensibles à l'amikacine. L'imipénème était remplacé dans la majorité des cas par l'amikacine ou une céphalosporine de troisième génération en cas de résistance.

Dans notre étude, les protocoles d'utilisation des antibiotiques reposaient sur une monothérapie : près de $65 \%$ des patients infectés étaient traités par imipénème dès qu'une infection était détectée et avant le résultat de l'antibiogramme. La dose du traitement antibiotique était conforme aux référentiels dans $95 \%$ des cas $[5,6]$.

Selon le résultat de l'antibiogramme, l'imipénème était prescrit dans $18 \%$ des infections nosocomiales, les céphalosporines de troisième génération dans $42 \%$, l'amikacine dans $24 \%$ et la vancomycine dans $7 \%$. Le choix de l'antibiotique était conforme aux résultats de l'antibiogramme.
La dose et la durée du traitement antibiotique étaient conformes aux référentiels dans $90 \%$ des cas $[5,6]$.

Face à ce problème réel, et dans le souci de dispenser des soins de qualité assurant aux patients à la fois l'efficacité thérapeutique et la sécurité, nous proposons quelques recommandations pratiques, notamment de renforcer les activités du CLIN pour l'investigation des infections nosocomiales. Cette unité sera chargée d'organiser la lutte contre les infections nosocomiales, de mettre en place des programmes de surveillance et de formation du personnel, voire de formation continue, aux pratiques de soins et d'hygiène, à la prescription des antibiotiques, et d'en établir des protocoles. Ces protocoles devront nous permettre d'améliorer le choix initial de l'antibiothérapie par la rédaction des recommandations en fonction des infections, de dresser une liste d'antibiotiques réservés à certaines indications et délivrés sur justification écrite.

L'implication de tous les personnels travaillant au sein de l'établissement dans la lutte contre les infections nosocomiales et la réalisation d'enquêtes en permanence portant sur l'incidence et la prévalence des infections nosocomiales ainsi que sur la prescription des antibiotiques doivent être menées dans chaque structure de santé sous l'égide du CLIN, qui est un instrument fondamental pour améliorer la qualité dans un hôpital.

\section{Conclusion}

Les infections nosocomiales constituent un indicateur de qualité des services et des établissements de santé dont dépend l'accréditation. Elles englobent deux grandes catégories de facteurs de risque. Les premiers sont intrinsèques aux malades et reflètent l'existence d'une immunodépression relative ou d'une pathologie responsable d'une sensibilité particulière à certaines infections. Les seconds sont des facteurs extrinsèques aux malades, directement ou indirectement liés aux interventions médicales et à l'environnement dans lequel celles-ci sont effectuées. Ces derniers peuvent être évités grâce à la surveillance et à la prévention.

La lutte contre les infections nosocomiales doit passer par une coordination avec les différents services médicaux (soins intensifs, médecine, pharmacie, chirurgie, laboratoire, etc.) sous l'égide du CLIN. La réduction de l'incidence et de la prévalence de ces infections passe non seulement par des mesures d'hygiène générales et spécifiques (gestes invasifs) dans l'hôpital mais aussi par une politique de prescription d'antibiotiques adaptée aux différentes situations et surtout ajustée en permanence à l'évolution des résistances bactériennes. L'usage approprié des antibiotiques, les modalités thérapeutiques et l'expérience clinique de chaque service conditionnent l'évolution de l'écologie bactérienne hospitalière.

\section{Références}

1. Brun-Buisson C, Girou E. Les infections nosocomiales : bilan et perspectives [Nosocomial infections: review and perspectives]. Médecine/sciences, 2000, 16(8-9):892-899.

2. Berche P, Gaillard JL, Simonet M, eds. Bactériologie - Bactéé ries des infections humaines [Bacteriology - Bacteria of human infections]. Paris, Médecine Sciences Publications, Collection PCEM Flammarion, 1991: 64-71.

3. Beaucaire G. Infections nosocomiales. Épidémiologie, critères du diagnostic, prévention, principes de traitement [Nosocomial infections. Epidemiology, diagnostic criteria, prevention, principles of treatment]. La Revue du Praticien, 1997, 47(2):201-209.
4. Centre de Coordination de la Lutte contre les Infections Nosocomiales de l'interrégion Nord (CCLIN Paris-Nord) (http:// www.cclinparisnord.org/Usagers/faq/FAQDream.htm, consulté le 11 mars 2012).

5. Portier $\mathrm{H}$ et al. Propositions thérapeutiques pour les principales infections nosocomiales [Therapeutic proposals for the main nosocomial infections]. In : Portier $\mathrm{H}$ et al. Antibioguide 2005 [Antibioguide 2005]. Bourgogne, Animé par la Commission des Anti-Infectieux de l'ARH (Agence Régionale de I'Hospitalisation) de Bourgogne, 2005:40-48.

6. Collège des Universitaires de Maladies Infectieuses et Tropicales (CMIT). Le POPI. Maladies infectieuses. Guide de 
traitement. Référence pour une bonne pratique médicale, $8^{e}$ édition [The POPI. Infectious diseases. Treatment guide. Reference forgood medical practice, 8th ed.]. Montmorency, 2M2, 2003.

7. Comité de I'antibiogramme de la Société Française de Microbiologie. Recommandations 2008 (http://www.sfmmicrobiologie.org/UserFiles/file/CASFM/casfm_2008.pdf, consulté le 25 mars 2012).

8. Fki $\mathrm{H}$ et al. Épidémiologie des infections nosocomiales dans les hôpitaux universitaires de Sfax : résultats de la première enquête nationale de prévalence de l'infection nosocomiale [Epidemiology of nosocomial infections in the university hospitals of Sfax: results of the first national point prevalence survey]. Revue Tunisienne d'Infectiologie, 2008, 2(1):22-31.

9. Sanchez-Velazquez LD, Ponce de Leon Rosales S, Sigfrido Rangel Frausto M. The burden of nosocomial infection in the intensive care unit: Effects on organ failure, mortality and costs. A nested case-control study. Archives of Medical Research, 2006, 37:370-375.

10. Maugat $\mathrm{S}$ et al. Ratio standardisé d'incidence : un indice de risque pour la surveillance des infections liées aux cathéters veineux centraux en réanimation adulte (réseau REACAT) dans l'inter-région nord [Standardized incidence ratio: a risk index for catheter-related infection surveillance in intensive care units (REACAT network) in Northern France]. Revue d'Épidémiologie et de Santé Publique, 2005, 53(S1):39-46.

11. Sligl W, Taylor G, Brindley PG. Five years of nosocomial Gram-negative bacteraemia in a general intensive care unit: Epidemiology, antimicrobial susceptibility patterns, and outcomes. International Journal of Infectious Diseases, 2006 , 10:320-325.

12. Dia NM et al. Résultats de l'enquête de prévalence des infections nosocomiales au CHNU de Fann (Dakar, Sénégal) [Results of a study on the prevalence of nosocomial infections at the National University Hospital, Dakar, Senegal]. Médecine et maladies infectieuses, 2008, 38(5):270-274.

13. Metinbas $\mathrm{S}$ et al. Prevalence and characteristics of nosocomia infections in a Turkish University Hospital. American Journal of Infection Control, 2004, 22:404-413.

14. Rigaux-Barry F, Blech MF. Enquêtes de prévalence des infections nosocomiales - Les résultats régionaux de la Lorraine de 1997 à 2000 [Prevalence surveys of nosocomial infections: results for the Lorraine Region from 1997 to 2000]. Hygiènes, 2003, 11(4):348-353.

15. Bridger JC. A study of nurses' views about the prevention of nosocomial urinary tract infections. Journal of Clinical Nursing, 1997, 6:379-387.

16. Agodi A et al. Active surveillance of nosocomial infections in urologic patients. European Urology, 2007, 51:247-254.

17. Christensen M, Jepsen OB. Reduced rates of hospital acquired UTI in medical patients. Prevalence surveys indicate effect of active infection control programmes. Journal of Hospital Infection, 2001, 47:36-40.

18. Halwani $\mathrm{M}$ et al. Cross-transmission of nosocomial pathogens in an adult intensive care unit: Incidence and risk factors Journal of Hospital Infection, 2006, 63:1-8.

19. LeeSC etal. Risk factors of mortality for nosocomial pneumonia: Importance of initial antimicrobial therap. International Journal of Clinical Practice, 2005, 59:39-45.

20. Michalopoulos A et al. Frequency, characteristics, and predictors of microbiologically documented nosocomial infections after cardiac surgery. European Journal of CardioThoracic Surgery, 2006, 29:456-460.

21. Baffoy N. Prévalence des infections nosocomiales chez les patients opérés [Prevalence of nosocomial infections in surgical patients]. Le Bulletin du CCLIN Paris-Nord, 2005, 1997, 7:6-8.

22. Materston $\mathrm{R}$ et al. Appropriate antimicrobial treatment in nosocomial infections: The clinical challenges. Journal of Hospital Infection, 2004, 56:142-149.

23. Besnier JM, Choutet P. Infections sur matériel étranger [Prosthetic device infections]. Médecine et Maladies Infectieuses, 1993, 23:765-767.

24. Vurma-Rapp $U$ et al. Mechanism of imipenem resistance acquired by three pseudomonas aeruginosa strains during imipenem therapy. European Journal of Clinical Microbiology \& Infectious Diseases, 1990, 9:580-587.

25. Nasnas R et al. Traitement des méningites à Pseudomonas et à Acinetobacter par l'amikacine par voie intrathécale [Treatment of Pseudomonas and Acinetobacter meningitis with intrathecal amikacin]. Médecine et maladies infectieuses, 1990, 20(11):573578 